\title{
Educational audiovisual text design: empirical evidence and new research perspectives
}

\author{
Giovanni Ganino
}

University of Ferrara, Italy, giovanni.ganino@unife.it, https://orcid.org/0000-0003-3973-9470

\begin{abstract}
The article presents a systematic analysis of international literature concerning the design of educational audiovisual texts at university level. The theme appears very important in light of the extensive use of these cognitive artefacts in flipped, blended, Massive Online Open Courses (MOOCs) teaching processes. This is even more important in the era of health emergency that has led to the use of audiovisual text as the main teaching medium at school and university. The aim of the work is to provide a contribution to research on educational technologies for the purpose of identifying new instructional design principles that support multimedia learning. The analysis highlighted new research directions, such as the significant role of the camera point of view in learning complex manual procedures, new design elements on the ways of representing the teacher and his/her communicative attitude, and the increasingly close relationship between educational sciences and neuroscience. The result may be useful, on the one hand, as a stimulus for an in-depth study of the new lines of research identified, by researchers on educational technologies, and on the other, for a more informed evidence-based use of audiovisual texts in teaching practices.
\end{abstract}

Keywords: Teaching media; open and distance learning; learning object; e-learning content; teaching methodology.

\section{Introduction}

The reference area of the design of audiovisual texts is that of didactic video or video-based learning, a research area that deals with theoretical and methodological aspects relating to the principles of knowledge construction through cognitive artefacts based on visual/audiovisual/audio/multimedia languages and network technologies, used with the aim of making learning processes meaningful. The study of these texts, used in the representation of factual, conceptual, procedural or metacognitive knowledge, in all subject areas is not a new phenomenon, but in recent years, with the spread of new didactic methods, flipped, blended, MOOCs, there has been a renewed interest in this technology. Not to mention the Covid-19 emergency that led to the use of audiovisual text as the main teaching medium. For this reason, it appears useful to identify scientific evidence related to the new design guidelines of these important educational resources.

\section{Background}

Numerous studies have shown how video can be effective in training processes if the rules of instructional design are respected: correct management of the cognitive load, enhancement of theories on multimedia learning, promotion of student involvement and active learning. All these points, especially the first two, are widely treated on a scientific level, but still do not find effective application in teaching practices, based on random or instrumental choices relating to the use of these resources (Ganino, 2018).

The theories on cognitive load and multimedia learning recall a long tradition of study, which can be placed within the discipline of instructional design (Clark \& Lyons, 2010; Mayer, 2009; Sweller, 1988; Sweller et al., 1998, 2019), according to which the enhancement of didactic content through videos or multimedia presentations made with the intention of reducing the extraneous cognitive load, increasing the relevant one and managing the intrinsic one improves learning outcomes. 
Regarding the promotion of involvement and attention, many researchers agree that audiovisual texts are the favourite educational resource among university students (Carmichael et al., 2018), thanks to the greater personalisation of the learning path and greater participation in problem-based learning educational processes allowed by these resources. For example, consider the possibility of learning from experts in the field, in a concrete and non-abstract way, according to a visual approach able to reduce the cognitive load necessary to mentally recall real situations or perform a process of mental animation to give concrete meaning to the processes, especially in STEM (Science, Technology, Engineering and Mathematics) subjects (Castro-Alonso et al., 2018). The involvement can have an impact on the behavioural, cognitive and emotional spheres, and the most easily measurable through web analytics tools is the first, the behavioural one, that is, the measurement of the frequency (of connection and for how long) and participation (carrying out self-assessment tests).

With regard to the enhancement of active learning, in addition to what has been stated in the previous point, there are solutions and methodologies that can promote the active role of the student and bring the mode of audiovisual use closer to the alphabetical-textual one. In particular, the two learning schemes, one based on immersion and the other on abstraction, can come together through the use of digital and multimedia languages, allowed, for example, by video annotation applications.

\section{Materials and methods}

The analysis of the literature presented was carried out according to a six-step process in accordance with the theory developed by Machi and McEvoy (2016), integrated, for point 3, with a protocol relating to the Prisma guidelines (Liberati et al., 2015).

Problem identification and analysis tool (points 1 and 2). Starting from the research objective - to provide a synthetic framework and critical analysis of the evidence emerging in the literature of audiovisual text design - the following question was formulated: Are there any guidelines that can help in the correct design of educational audiovisual texts?

Identification, collection and organisation of literature (point 3). For the identification and selection of scientific literature, primary and secondary studies, a series of databases were queried through the Library System of the University of Ferrara (cerc@unife, EBSCO Discovery Service):

- the research was carried out in the months of March 2020-November 2020;

- the research was limited to articles published in English over the previous 10 years;

- Elsevier Science Direct, ISI Web of Science, JSTOR, Open Dissertations, Scopus, SpringerLink, Wiley Online Library and Rivisteweb-Il Mulino/Carocci databases were consulted. The search was refined with the Google Scholar query.

A series of peer-reviewed academic publications with links to the full text were identified through the following search keywords and related Boolean operators: instructional video OR video lectures OR educational videos OR online lecture AND (University OR higher education OR college). One thousand six hundred and seventy-five studies were identified (see Fig. 1); 54 reviews and research reports were deleted from these. The research was perfected through selection of the aggregated contributions according to the following categories: education, learning, teaching methods, educational technology, higher education, teaching, online education, educational films, e-learning, distance education, instructional films, learning strategies, blended learning, flipped classroom. This process identified 1210 relevant articles. The subsequent review process (reading of the abstract and then of the entire text) identified 21 articles.

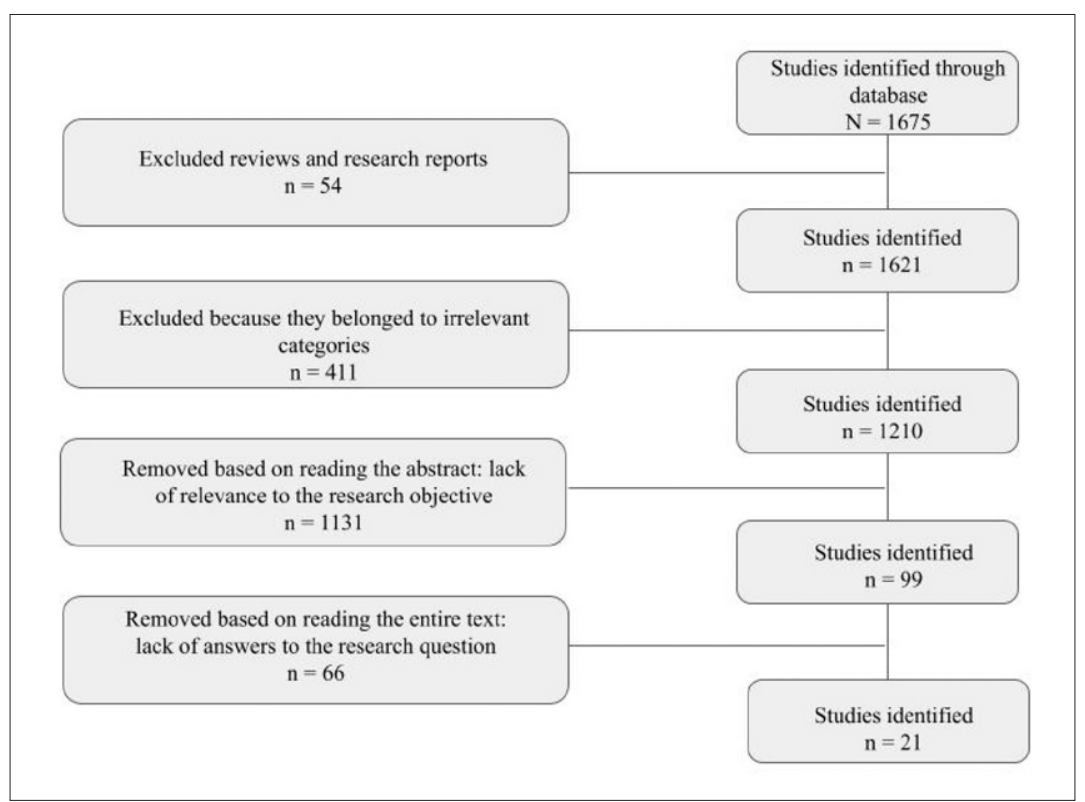

Fig. 1 Flow chart of the studies included in the review 


\section{Educational audiovisual text design: empirical evidence and new research perspectives}

Giovanni, G.

\section{Results}

Data analysis and critique of the identified contents (points 4, 5 and 6 of the six-phase process) were carried out with respect to the research question. We have grouped them into three research areas - the significant role of the subjective point of view of the camera, new design elements relating to the mode of representation of the teacher and his/her communicative attitude, the increasingly close relationship between educational sciences and neuroscience - each linked to a specific multimedia principle (see Table 1).

Table 1. Summary of the data extrapolated from the identified studies

\begin{tabular}{|c|c|c|c|}
\hline & Authors (year) & Research area & Focus \\
\hline 1 & Lyons et al. (2012) & Representation of the teacher & $\begin{array}{l}\text { Social presence signals reduce perceived learning } \\
\text { for students with low technological knowledge; they } \\
\text { increase learning in online courses (students with } \\
\text { greater digital skills) }\end{array}$ \\
\hline
\end{tabular}

3 Garland and Sanchez (2013)

4

Fiorella and Mayer (2016) Representation of the teacher Jannin et al. (2017)

Video and subjective point of view (over the shoulder)

Close relationship between educational sciences and neuroscience

Representation of the teacher

Representation of the teacher

Representation of the teacher

Egocentric (subjective) versus heterocentric (objective) point of view and mental rotation

Fattorini and Paoletti Representation of the teacher (2017)

van Wermeskerken et al. Representation of the teacher (2017)

Boucheix et al. (2018)

Wilson et al. (2018);

Chen et al. (2018)

van Merriënboer and Kirschner (2018)

Sweller et al. (2019)

Anmarkrud et al. (2019)

Schmidt et al. (2020)

Wang et al. (2020)
Mixed camera point of view (subjective, objective)

Representation of the teacher sciences and neuroscience

Close relationship between educational sciences and neuroscience

Close relationship between educational sciences and neuroscience sciences and neuroscience

Subjective point of view of the camera

Close relationship between educational sciences and neuroscience
Principle of gestures and lexical recovery by the teacher

Procedural learning about knot tying

Cognitive load and environmental factors

Use of talking head, Khan style drawings and brevity lead to greater involvement

Sense of social presence and positive effect on information memorisation, attention and interest

Principle of the image: strategic use of the teacher's face (alternating with the visual message) promotes a sense of social presence without determining an extraneous cognitive load

Hand movement and relevant information

Procedural learning about five surgical sutures

Principle of gestures and multimedia message enhancement

People learn better from a video lesson when the onscreen instructor shifts his/her gaze between the audience and board

Procedural learning: catheter insertion in the human body

Presence increases the student's sense of satisfaction even if it compromises learning due to the division of attention

Close relationship between educational New working memory modes

Cognitive load and 4C/ID four-component model

Discipline progress in terms of new educational effects and future research directions

Nine-level Likert scales to self-measure overall cognitive load or intrinsic, extraneous or relevant loads

Procedural learning: laparoscopy and knot suturing

Presence of the teacher and cognitive load: joint use of subjective measurement and objective measurement based on the EEG

Significant value of the gaze and dynamic drawing increase the sense of social collaboration 


\section{Educational audiovisual text design: empirical evidence and new research perspectives}

Giovanni, G.

\subsection{The camera point of view: perception and learning}

Several studies identified have investigated the role of the subjective or third-person point of view of the camera in learning procedural skills. This point of view appears useful in creating effective educational videos in terms of reducing procedural learning time and the likelihood of making mistakes in carrying out the task. Through the use of the subjective point of view, Garland and Sanchez (2013) have verified improvements in knot-tying skills and Jannin et al. (2017) have shown improvements by medical students in the insertion of medical sutures.

Boucheix et al. (2018) investigated the role of subjective point of view in learning a complex manual procedure in the medical field - the insertion of a urinary catheter into the human body simulated by a manikin. In the research involving 43 French students divided into three experimental groups, the procedure (consisting of 25 phases, divided into five modules) was shown in three different videos of the same duration: a) from a frontal perspective, b) from a teacher shoulder perspective, or in first person and c) from a mixed perspective. The results were better for the three experimental groups than for the control group, confirming the benefits of video in procedural learning. The students who were given the video with the dual point of view scored higher than the other groups. The method of construction of the instructional video according to the double point of view procedure was also tested in a work by Schmidt et al. (2020) related to a self-training teaching activity for medical students (University of Heidelberg in Germany): learning of the laparoscopic suture and the relative tying of the knot according to the C-loop technique. The experimentation with the presence of blinded evaluators involved the presence of two groups subjected to: a) watching a video made through the combination of a double point of view, in first person (view of hands, instruments and movement of the forearm) and in third person (the endoscopic image) and b) watching a video exclusively composed of the endoscopic view. Although the participants of both groups perceived the first-person perspective as useful for learning new laparoscopic skills, the average training time to reach protocol competence did not show any significant differences between the two groups. The contradiction between objective performance and subjective perception by the participants in this study may have been influenced by multiple factors. Consider, for example, how research (in Schmidt et al., 2020) recommends the use of third-person images for learning basic laparoscopic skills and first-person images for learning more complex skills (also supplemented by good initial psychomotor skills). Discrepancy in results should, therefore, be better assessed in future research, possibly using a more homogeneous study population in terms of laparoscopic experience.

\subsection{Mode of instructor's representation and his/her communicative attitude}

A much discussed topic concerns the choice relating to the presence/absence of the teacher within the audiovisual texts based on two main reference theories, that of social presence and that of the increase in cognitive load due to divided attention.

The research activities identified, relating to the first theory, consider social signals as useful for creating greater involvement, in terms of motivation and collaboration of the student: the presence of the teacher increases the sense of satisfaction of the student, who, for this reason, believes to have learned more (Kizilcec et al., 2015; Lyons et al., 2012; Wang et al., 2020; Wilson et al., 2018) and determines to show more interest and have interaction with the course content (Guo et al., 2014). Kizilcec and colleagues compared the constant inclusion of a talking head in the video with a moderate presence and discovered that, although there were no significant differences in terms of learning performance, the students preferred the constant presence of the teacher's face. The research activities related to the cognitive load theory consider the image of the instructor as a cause of split attention: the student is distracted from the didactic content by the presence of signals such as gestures, eye contact and facial expressions, as highlighted by several eyepiece-tracking studies (Kizilcec et al., 2014; Wang et al., 2020). Other experiments have provided empirical evidence to support this theoretical assumption, as they did not reveal any obvious advantage from the presence of the instructor (Kizilcec et al., 2014) and highlighted obstacles to learning performance (Wilson et al., 2018).

Other recurring themes identified in the review concern the communicative attitude of the teacher in terms of the functional use of gestures, gazes, hand movement and dynamic drawing. As stated by Fattorini and Paoletti (2017) is important to investigate the role of illustrative gestures whose characteristics in terms of the functions of clarification, extension or highlighting of the content, identified by Poggi and Caldognetto (1997), can influence the communicative dynamics of the didactic message. The gestures that signal important information, organise them and explain them can have significant value in didactic processes, both for the student in terms of greater propensity to remember and for the speaker in terms of better expressive capacity and reduction of pauses associated with lexical recovery difficulties (Cook et al., 2012). Fattorini and Paoletti paid attention to the use of gestures through making a distinction between significant and insignificant gestures and finding the connection between gestures and spoken speech, not only as relevant elements for student learning and motivation, but also as possible sources of extraneous cognitive load, when exclusively expressive and non-informative.

In relation to the gaze, during the video lesson, the instructor can keep his/her gaze fixed or change direction on the students or visuals. Research indicates that students learn best when the instructor alternates his/her gaze. In this sense, the act of looking has a significant function as it suggests to the student where to direct his/her attention and how to avoid divided attention phenomena (van Wermeskerken et al., 2017). The use of transparent boards appears useful in the attribution of significant value to gazes since they enhance both the paradigm of the social agency theory, maintaining continuous visual contact (Mayer et al., 2020), and the signalling principle by giving indications with the shifts of the gaze. 


\section{Educational audiovisual text design: empirical evidence and new research perspectives}

Giovanni, G.

Hand movements can provide relevant information continuously and at the right time, avoiding cognitive waste caused by irrelevant and distracting non-verbal information (Boucheix \& Forestier, 2017; Fiorella \& Mayer, 2016). The movement of the hands allows you to direct attention towards relevant processes and concepts in order to improve understanding, thus becoming significant events. The role of the hand in composing the didactic message was also investigated by Mayer et al. (2020) in their study on the principle of dynamic drawing, a principle connected to the social agency theory (Mayer, 2014) and embodiment theory (Robbins \& Aydele, 2009): seeing graphic forms in their temporal and spatial composition is a signal that favours a sense of social collaboration and, as a consequence, deeper learning; in the perspective of the embodiment theory, the hand of the instructor in action triggers a sense of self-referentiality, thus favouring the student's cognitive processing.

\subsection{The contribution of neuroscience to multimedia learning}

The research activities identified in the relationship between neuroscience, cognitive psychology and educational sciences seem to make an important contribution to the education design sector. Regarding cognitive load measurements, many researchers in the field of education have adopted subjective techniques.

The most used method involved the use of nine-level Likert scales to measure the overall cognitive load or intrinsic, extraneous or relevant loads (Anmarkrud et al., 2019). The need to overcome the limits of subjective measurements has introduced alternative methods in the context of cognitive neuroscience, based on physiological assessments of the electrical oscillation activities of the brain. With electroencephalography (EEG) used in authentic learning contexts, an attempt was made to verify the meaning of brain oscillations in the analysis of cognition and attention mechanisms. The studies cited in the article by Wang and colleagues identified two different electrical frequencies in relation to cognitive processes; the active processing of information in the working memory occurs through brain oscillations in the alpha and theta frequency bands: the power of the theta band increases and that of the alpha band decreases in the presence of a greater cognitive demand. More generally, the progress of the last 20 years of cognitive load theory and the future directions of research in this area can be observed in a study by Sweller et al. (2019). Progress relates to:

1.the strengthening of its theoretical basis through a more meaningful classification of didactic information by inserting the studies of human cognitive architecture in the area of evolutionary psychology (Sweller, 2016);

2. the application of the theory of cognitive load on long-term educational activities, for example, to the design of courses or entire curricula according to the four-component model 4C/ID, characterised by high interactivity in the didactic processes aimed at developing professional skills or competences (van Merriënboer \& Kirschner, 2018) and

3. the identification of new cognitive load effects: instructive effects (of self-explanation, imagination, isolated elements, collective working memory and the effect of human movement) and compound effects, that is, effects that modify the characteristics of other effects, defined as simple, of cognitive load (element interactivity, expertise reversal, guidance fading, transient information, self-management, self-explanation, imagination, isolated elements, collective working memory and human movement effect).

Future directions of cognitive load research are indicated in the following studies: new ways of functioning of working memory whose ability to store information would not be fixed, but would be variable, thus overcoming the 'bottleneck' of learning (Chen et al., 2018); the students' ability to self-regulate their own learning resources; the influence on the cognitive load of environmental factors such as stress, emotions and cognitive insecurity (Choi et al., 2014), useful in cases where they are an integral part of the learning task (therefore falling within the intrinsic cognitive load), for example, insecurity due to the need for rapid decision-making processes with an uncertain outcome; a fourth line of research concerns the effect of human movements, gestures, gaze and the movement of the index finger with the function of guiding and directing the gaze on the available working memory resources and on the cognitive load (Sweller et al. 2019).

\section{Discussion}

Evidence shows how audiovisual resources can have a significant impact on learning as long as they are created in compliance with precise theoretical paradigms recognised by the scientific community. The review work performed highlights the expansion of the didactic video, thanks to greater design evidence - in the significant role of the subjective point of view used in the videos for the learning of procedural knowledge and the representation modes of the teacher as well as his/her communicative attitude - and a closer link between educational sciences and neuroscience. The significant role of the point of view of the camera in the representation of the procedural abilities - objective or in third person, subjective or in first person, a combination of the two - appears to be very useful in the creation of effective didactic videos in terms of reducing both learning time and the probability of making mistakes in performing the task. Reference scientific theories in this area are the theory of mirror neurons according to which the integrated use of different points of view increases the activation of the neuronal system, already triggered by the dynamic observation of manual procedures (a series of research on the vision of manual procedures according to a dynamic and subjective perspective that have shown improvements in terms of memorisation and subsequent recall of information), some so-called self-reference psychological factors, which determine greater effectiveness in remembering the processes observed subjectively, and the embodiment theory according to which people 
think and learn with their own body as well as with their mind (Robbins \& Aydele, 2009). According to this last theory, the first-person perspective causes a state of self-reference (what the observers see refers to themselves), which determines a stronger link between the observer and what is observed, and therefore a greater propensity towards deep learning. Therefore, the optimal attitude of the students in a situation of learning manual procedures is based on the alternation between looking at the instructor (frontal point of view or in the third person) and thinking of putting into practice the observed procedure (subjective point of view or in the first person). The alternation of the two points of view, objective and subjective, and the simplification/organisation of the observed events through their analytical analysis determines a plausible simulation of the media-based learning experience that more easily determines learning (in terms of transfer) of complex manual procedures. It is important to underline how the video representation of the procedures observed can benefit from the use of the point-ofview technique (objective and subjective), but only if this brings about a real benefit in terms of profound learning of skills. This may mean that some processes require a representation based on several points of view, while other processes based on a single point of view, sometimes in the first person and others in the third. In line with the positive effect of space-time alignment with respect to perceptual importance and conceptual relevance to the requirements of the procedure (Lowe et al., 2010): in practice, it is suggested how the staging of the didactic video, in terms of analytical decomposition and points of view adopted, depends on the appropriateness of the task to be learned.

Many studies on the design of multimedia educational resources are focusing on the mode of representation of the teacher, his/her presence or absence, and his/her communicative attitude. Most online video formats present an instructor on the screen to improve students' involvement and their processing of information, but there is conflicting evidence in this area. Unless it is supported by the multimedia design principles underpinning the learning process, the presence of the instructor in the video can compromise understanding, even if students prefer this format in the belief of achieving better results. This highlights a contrast between perception/enjoyment and learning (studies straddling educational sciences and neuroscience can provide useful information on this point). Regarding the communicative attitude of the teacher, it has been seen how gestures play an important role for students who can remember the contents of the lesson if supported by redundant, integrative or emotional gestures with respect to the verbal statement. However, gestures can also have an effective role for teachers in terms of reducing the cognitive demands on their working memory, but it is not clear whether these benefits depend on the properties of the gesture, rhythmic movement or the vehicle for representing meaning (Cook et al., 2012). It is important to consider such expressive elements, together with the gaze, hand movements and dynamic design, as a single didactic communication system in the direction of conscious use of social signals that can promote a sense of partnership and the cognitive processing of the student, thus leading to profound learning.

Progress in research in the design area proceeds hand in hand with the evolution of the scientific principles related to the link between educational sciences and neuroscience. An example is the progress in cognitive load theory, which has led to the identification of new principles useful for didactic design and the development of new objective cognitive load measurements through the use of physiological nature and neuroimaging techniques such as magnetic resonance, EEG techniques or eye tracking. Measurements with techniques that can evaluate the role of the different electrical frequencies in authentic learning situations on cognitive processes, through EEG, appear to be particularly interesting. This latter aspect is not only functional to the objective measurement of the triple dimension of cognitive load, but could also contribute to the design of audiovisual texts in general and to the design of adaptive brain-computer interfaces able to adapt the instructions provided in virtual environments to the students on the basis of their brain activity.

\section{Conclusions}

The omnipresence of educational audiovisual texts has determined a renewed interest in research in this area. The most interesting and innovative scientific contributions concern the role of the subjective image, new principles relating to the way the instructor is represented in the video as well as his/her communicative attitude, and the increasingly close relationship between educational sciences and neuroscience. All of the identified principles must be further verified through more specific meta-analysis and experimental research. Finally, the work carried out shows that, in order to obtain positive effects on learning, it is necessary to design educational resources based on the complementary integration of evidence in the perceptual, cognitive and multimedia design fields.

\section{References}

Anmarkrud, O., Andresen, A., \& Bråten, I. (2019). Cognitive Load and Working Memory in Multimedia Learning: Conceptual and Measurement Issues. Educational Psychologist, 54 (2), 61-83. https://doi.org/10.1080/00461520.2018.1554484

Boucheix, J.M., \& Forestier, C. (2017). Reducing the transience effect of animations does not (always) lead to better performance in children learning a complex hand procedure. Computers in Human Behavior, 69, 358-370. https://doi. org/10.1016/j.chb.2016.12.029

Boucheix, J.M., Gauthier, P., Fontaine, J., \& Jaffeux, S. (2018). Mixed camera viewpoints improve learning medical hand procedure from video in nurse training? Computers in Human Behavior, 89, 418-429. https://doi.org/10.1016/j. chb.2018.01.017 


\section{Educational audiovisual text design: empirical evidence and new research perspectives}

Giovanni, G.

Carmichael, M., Reid, A.K., \& Karpicke, J. D. (2018). Assessing the Impact of Educational Video on Student Engagement, Critical Thinking and Learning: The Current State of Play (White Paper). Thousand Oaks, CA: SAGE Publishing, Inc. Retrieved from, https://education.report/Resources/Whitepapers/aa1c324d-7d2e-4dd5-916a-e146ef5e2884_hevideolearning.pdf

Castro-Alonso, J.C., Ayres, P., Wong, M., \& Paas, F. (2018). Learning symbols from permanent and transient visual presentations: Don't overplay the hand. Computers \& Education, 116, 1-13. https://doi.org/10.1016/j. compedu.2017.08.011

Chen, O., Castro-Alonso, J. C., Paas, F., \& Sweller, J. (2018). Extending cognitive load theory to incorporate working memory resource depletion: evidence from the spacing effect. Educational Psychology Review, 30(2), $483-501$. https://doi.org/10.1007/s10648-017-9426-2

Choi, H. H., van Merriënboer, J. J. G., \& Paas, F. (2014). Effects of the physical environment on cognitive load and learning: towards a new model of cognitive load. Educational Psychology Review, 26, 225-244. https://doi.org/10.1007/ s10648-014-9262-6

Clark, R., \& Lyons, C. (2010). Graphics for Learning: Proven Guidelines for Planning, Designing, and Evaluating Visuals in Training Materials. San Francisco: Pfeiffer.

Cook, S.W., Yip, T.K., \& Goldin-Meadow, S. (2012). Gestures, but not meaningless movements, lighten working memory load when explaining math. Language and cognitive processes, 27(4), 594-610. http://doi.org/10.1080/01690965.20 11.567074

Fattorini, R., \& Paoletti, G. (2017). Mettiamoci la faccia. L’uso dei Talking Head nelle lezioni online. Form@re. Open Journal per la formazione in rete, 17(1), 217-227. http://dx.doi.org/10.13128/formare-20163

Fiorella, L., \& Mayer, R.E. (2016). Effects of observing the instructor draw diagrams on learning from multimedia messages. Journal of Educational Psychology, 108(4), 528-546. https://doi.org/10.1037/edu0000065

Ganino, G. (2018). Video didattica. Comunicazione visiva, apprendimenti multimediali e processi cognitivi. Lecce-Brescia: PensaMultiMedia.

Garland, T.B., \& Sanchez, C.A. (2013). Rotational perspective and learning procedural tasks from dynamic media. Computers and Education, 69, 31-37. https://doi.org/10.1016/j.compedu.2013.06.014

Guo, P. J., Kim, J., \& Rubin, R. (2014). How video production affects student engagement: An empirical study of mooc videos. In Proceedings of the First ACM Conference on Learning@ Scale Conference, ACM, 41-50. https://doi. org/10.1145/2556325.2566239

Jannin, L., Ganier, F., \& DeVries, P. (2017). Effet du point de vue de présentation des instructions et de l'aptitude à la rotation mentale sur l'apprentissage d'un geste technique. Conférence EPIQUE, Dijon, France.

Kizilcec, R.F., Bailenson, J.N., \& Gomez, C.J. (2015). The instructor's face in video instruction: evidence from two largescale field studies. Journal od Educational Psychology, 107(3), 724-739. https://doi.org/10.1037/edu0000013

Kizilcec, R.F., Papadopoulos, K., \& Sritanyaratana, L. (2014). Showing face in video instruction: effects on information retention, visual attention, and affect. Proceedings of the 32 nd annual ACM conference on Human factors in computing system, ACM, 2095, 2102. https://doi.org/10.1145/2556288.2557207

Liberati, A., Altman, D. G., \& Tetzlaff, J. (2015). PRISMA Statement per il reporting di revisioni sistemiche e meta-analisi degli studi che valutano gli interventi sanitari: spiegazione ed elaborazione. Evidence, 7(6), 1-36.

Lowe, R., Schnotz, W., Rasch, T. Aligning affordances of graphics with learning task requirements. Applied Cognitive Psychology, 25: 452-459 (2011), Published online 21 May 2010 in Wiley Online Library (wileyonlinelibrary.com) https://doi.org/10.1002/acp.1712

Lyons, A., Reysen, S., \& Pierce, L. (2012). Video lecture format, student technological efficacy, and social presence in online courses. Computers in Human Behavior, 28(1), 181-186. https://doi.org/10.1016/j.chb.2011.08.025

Machi, L.A., \& McEvoy, B.T. (2016). The literature review: Six steps to success. Thousand Oaks, CA: Corvin Press.

Mayer, R.E. (2009). Multimedia learning, New york: Cambridge University Press.

Mayer, R.E. (2014). Multimedia instruction. In J. M. Spector, M. D. Merrill, J. Elen, \& M. J. Bishop (Eds.). Handbook of Research on Educational Communications and Technology, 385-399. New York: Springer.

Mayer, R.E., Fiorella, L., \& Stull, A. (2020). Five ways to increase the effectiveness of instructional video. Education Tech Research Dev, 68(3), 837-852. https://doi.org/10.1007/s11423-020-09749-6

Poggi, I., \& Caldognetto, E.M. (1997). Mani che parlano. Gesti e psicologia della comunicazione. Padova: Unipress.

Robbins, P., \& Aydele, M. (2009). A short primer on situated cognition. In P. Robbins \& M. Aydede (Eds.), The Cambridge handbook of situated cognition. New York: Cambridge University Press. 
Schmidt Mona W., Kowalewski, K.F., Trent, S.M., Benner Laura, Müller-Stich Beat P., \& Nickel, F. (2020). Self-directed training with e-learning using the first-person perspective for laparoscopic suturing and knot tying: a randomised controlled trial. Surgical Endoscopy, 34, 869-879. https://doi.org/10.1007/s00464-019-06842-7

Sweller, J. (1988). Cognitive Load during Problem Solving: Effects on Learning. Cognitive Science, 12(2), $257-285$. https://doi.org/10.1016/0364-0213(88)90023-7

Sweller, J. (2016). Cognitive load theory, evolutionary educational psychology, and instructional design. In D. Geary, \& D. Berch (Eds.), Evolutionary perspectives on child development and education, 291-306. https://doi.org/10.1007/9783-319-29986-0_12

Sweller, J., van Merriënboer, J.J.C., \& Paas, F.G. (1998). Cognitive Architecture and Instructional Design. Educational Psychology Review, 10, 251-296. https://doi.org/10.1023/A:1022193728205

Sweller, J., van Merriënboer, J.J.C., \& Paas, F.G. (2019). Cognitive Architecture and Instructional Design: 20 Years Later. Educational Psychology Review, 31, 261-292. https://doi.org/10.1007/s10648-019-09465-5

van Merriënboer, J.J.G., \& Kirschner, P.A. (2018). 4C/ID in the context of instructional design and the learning sciences. In F. Fisher, C.E. Hmelo-Silver, S.R. Goldman, \& P. Reimann (Eds.), International handbook of the learning sciences (pp. 169-179). New York: Routledge. https://doi.org/10.4324/9781315617572-17

van Wermeskerken, M., \& van Gog, T. (2017). Seeing the instructor's face and gaze in demonstration video examples affects attention allocation but not learning. Computer Education 113, 98-107. https://doi.org/10.1016/j. compedu.2017.05.013

Wang, J., Antonenko, P., Keil, A., \& Dawson, K. (2020). Converging Subjective and Psychophysiological Measures of Cognitive Load to Study the Effects of Instructor-Present Video. Mind, Brain, and Education, 14 (3), 279-291. https:// doi.org/10.1111/mbe.12239

Wilson, K. E., Martinez, M., Mills, C., D’Mello, S., Smilek, D., \& Risko, E. F. (2018). Instructor presence effect: Liking does not always lead to learning. Computers \& Education, 122, 205-220. https://doi.org/10.1016/j.compedu.2018.03.011 